\title{
Australian experience of a modified schedule of FOLFOX with high activity and tolerability and improved convenience in untreated metastatic colorectal cancer patients
}

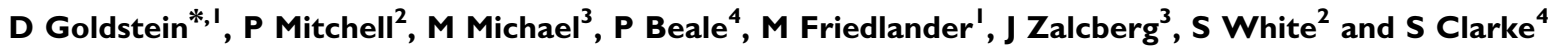 \\ 'Department of Medical Oncology, Prince of Wales Hospital, Randwick, Sydney, NSW 2031, Australia; '2Department of Medical Oncology, Austin and \\ Repatriation Medical Centre, Melbourne, Australia; ${ }^{3}$ Department of Medical Oncology, Peter MacCallum Cancer Centre, Melbourne, Australia; \\ ${ }^{4}$ Department of Medical Oncology, Royal Prince Alfred Hospital, Sydney, Australia
}

This study determined the efficacy and safety of a modified FOLFOX regimen that improved patient convenience without compromising oxaliplatin dose intensity. A total of 62 patients with previously untreated metastatic colorectal cancer were enrolled to receive, entirely as outpatients, 2-weekly cycles of oxaliplatin $100 \mathrm{mg} \mathrm{m}^{-2}$ i.v. over $2 \mathrm{~h}$, together with leucovorin $400 \mathrm{mg} \mathrm{m}^{-2}$ over $2 \mathrm{~h}$, 5-fluorouracil (5-FU) $400 \mathrm{mg} \mathrm{m}^{-2}$, bolus, followed by a 46 -h infusion of 5-FU at $2.4 \mathrm{~g} \mathrm{~m}^{-2}$. Treatment was given until progression or unmanageable toxicity. In all, 6 I patients received $\geqslant$ one oxaliplatin dose and a median of I I treatment cycles (range I -20 cycles); 22 (36\%) reported grade 3/4 neutropenia and 13 patients (21\%) experienced grade 3 neurotoxicity; 16 patients (26\%) discontinued treatment due to disease progression or death, 15 (25\%) due to neurotoxicity and six (10\%) due to haematological toxicity. Of the 56 eligible patients, complete or partial responses were observed in 29 or 52\% (95\% confidence interval 38-65\%). Median progression-free survival was 8.2 months $(7.1$-9.9) and median overall survival was 18.7 months (14.0-23.4). In our experience, a modified schedule of FOLFOX improves convenience without compromising efficacy or toxicity.

British Journal of Cancer (2005) 92, 832-837. doi:I0.1038/sj.bjc.6602426 www.bjcancer.com

Published online I March 2005

(c) 2005 Cancer Research UK

Keywords: colorectal cancer; dose intensity; 5-fluorouracil; neurotoxicity; oxaliplatin

Oxaliplatin unlike cisplatin has demonstrated activity against colon carcinoma cell lines in vitro and has also shown synergistic activity in experimental models (Raymond et al, 1997). Several large randomised trials have confirmed the efficacy of the oxaliplatin/5-fluorouracil (5-FU)/leucovorin (LV) combination as first-line therapy in metastatic colorectal cancer. In De Gramont's et al (2000) phase III trial, FOLFOX4 was significantly superior to the infusional regimen, LV5FU2, in response rate and progressionfree survival (PFS) and the combination of chronomodulated 5-FU/LV and oxaliplatin produced a significantly higher response rate and superior PFS to the 5-FU/LV regimen alone (Giacchetti et al, 2000). In a recent three-arm study in 796 untreated patients with metastatic colorectal cancer (Goldberg et al, 2004), FOLFOX4 was significantly superior to the irinotecan and bolus 5-FU/LV combination (IFL) and a third regimen of oxaliplatin plus irinotecan (IROX) in response rate, time to progression and overall survival, and induced fewer Grade 3 or 4 toxicities.

The original FOLFOX4 regimen, however, does have drawbacks for patients, as it requires intravenous treatment in hospital for

\footnotetext{
*Correspondence: Dr D Goldstein: E-mail: d.goldstein@unsw.edu.au Interim results of this study were presented at the Annual Meeting of the American Society of Clinical Oncology (ASCO), San Francisco, May 200 I Revised I November 2004; accepted 2 I December 2004; published online I March 2005
}

48-h every 2 weeks. Several variations of this regimen, incorporating oxaliplatin dose intensification or simplification of the LV/5-FU schedule, have been evaluated in clinical trials.

A retrospective analysis by Maindrault-Goebel et al (2000) of three phase II studies, in previously treated patients, using three different FOLFOX regimens showed that increased oxaliplatin dose intensity ( $>85 \mathrm{mg} \mathrm{m}^{-2}$ ) improved response rates and PFS without compromising tolerability. One of the more effective regimens, FOLFOX6, utilised an oxaliplatin dose of $100 \mathrm{mg} \mathrm{m}^{-2}$ on day 1 in combination with a simplified bimonthly 5-FU/LV schedule (LV $400 \mathrm{mg} \mathrm{m}^{-2}$ on day 1 only, followed by 5 -FU bolus $400 \mathrm{mg} \mathrm{m}^{-2}$, then $2400-3000 \mathrm{mg} \mathrm{m}^{-2}$ as a $46-\mathrm{h}$ infusion). In the phase II trial with FOLFOX6 in pretreated patients, a response rate of $27 \%$ was achieved, but with significant grade 3 or 4 neutropenia (24\%) and neurotoxicity of $16 \%$ (Maindrault-Goebel et al, 1999).

In this Australian phase II study in untreated patients with metastatic colorectal cancer, we also sought to simplify the treatment delivery schedule of the active FOLFOX4 regimen without compromising dose intensity. We eliminated the 2 nd day bolus dose of 5-FU, increased the dose of oxaliplatin to $100 \mathrm{mg} \mathrm{m}^{-2}$ and increased the 5 -FU infusion to $46 \mathrm{~h}$, similar to FOLFOX6, and also utilised disposable pumps to allow an entirely outpatient treatment. In the algorithm for dose reductions due to haematological toxicity, we chose, in contrast to FOLFOX6, to preferentially dose-reduce 5-FU rather than oxaliplatin, aiming to maximise oxaliplatin dose intensity. In FOLFOX6 both the 
oxaliplatin dose was reduced to $75 \mathrm{mg} \mathrm{m}^{-2}$ and 5 -FU infusion to $2000 \mathrm{mg} \mathrm{m}^{-2}$ without altering the bolus dose of 5 -FU. In our schema the first dose adjustment was elimination of the bolus $5-\mathrm{FU}$, the second was reduction of the continuous infusion of 5FU and only then reduction of oxaliplatin. The primary objective of our study was to determine the objective response rate of this modified FOLFOX6 regimen. Our secondary objective was to assess the qualitative and quantitative toxicities of this regimen.

\section{PATIENTS AND METHODS}

\section{Patients}

Patients with a histologically confirmed diagnosis of advanced adenocarcinoma of the colon or rectum were eligible to be entered into the trial provided they were aged $\geqslant 18$ years, had measurable disease (defined as at least one lesion with at least one diameter $\geqslant 2 \mathrm{~cm}$ ), ECOG performance status (PS) of $0-2$ and had completed adjuvant treatment 6 or more months prior to study entry. Patients who received prior adjuvant treatment with oxaliplatin were not eligible. To be included, patients also had to have a serum creatinine $\leqslant 160 \mu \mathrm{moll}^{-1}$, bilirubin $\leqslant 1.5 \times$ the upper limit of normal (ULN), ALT $\leqslant 4 \times$ ULN, an absolute neutrophil count (ANC) $\geqslant 1.5 \times 10^{9} 1^{-1}$, platelets $\geqslant 100 \times 10^{9} 1^{-1}$ and peripheral neuropathy $\leqslant$ NCI Common Toxicity Criteria (CTC) grade 1 . Patients were required to provide signed informed consent.

Patients were excluded if they had received prior therapy for advanced colorectal cancer, any uncontrolled infection, a history of myocardial infarction within the previous 6 months or current clinical evidence of congestive heart failure or unstable angina, a history of any other cancer (except nonmelanoma skin cancer or carcinoma in situ of the cervix) unless in complete remission and off all therapy for that cancer for at least 5 years, central nervous system metastases, or were pregnant or lactating or could not provide informed consent. Men and women of reproductive potential had to agree to use effective contraception.

\section{Study design}

The study protocol was approved by each of the participating institutions' ethics committees and was conducted according to the Declaration of Helsinki. This was an open-label multicentre trial to assess the response rate and quantitative and qualitative toxicities of a modified schedule of FOLFOX.

Patients were registered by the investigator faxing the registration form to the sponsor Sanofi Synthelabo and then treatment could commence.

\section{Chemotherapy}

Treatment was given entirely as an outpatient. On day 1 of each 2 -week cycle, patients received oxaliplatin (Eloxatin ${ }^{\circledR}$ supplied by Sanofi Synthelabo) $100 \mathrm{mg} \mathrm{m}^{-2}$ given as an intravenous infusion over $2 \mathrm{~h}$ together with $\mathrm{LV} 400 \mathrm{mg} \mathrm{m}^{-2}$ over $2 \mathrm{~h}$ and then $5-\mathrm{FU}$

5-FU bolus $400 \mathrm{mg} \mathrm{m}^{-2}$
\begin{tabular}{|c|c|}
\hline $\mathrm{LV}$ & \\
$400 \mathrm{mg} \mathrm{m}^{-2}$ & $5-\mathrm{FU}$ infusion via disposable pump \\
& $2.4 \mathrm{~g} \mathrm{~m}^{-2}$ over $46 \mathrm{~h}$ \\
\hline $\begin{array}{c}\text { Oxaliplatin } \\
100 \mathrm{mg} \mathrm{m}^{-2}\end{array}$ & \\
\hline $0 \mathrm{~h}$ & \\
\hline \multicolumn{2}{c}{$\mathrm{h}$}
\end{tabular}

Figure I Chemotherapy regimen.
$400 \mathrm{mg} \mathrm{m}^{-2}$ as a bolus injection followed by a $46-\mathrm{h}$ continuous infusion of 5-FU at a total dose of $2.4 \mathrm{~g} \mathrm{~m}^{-2}$ (Figure 1). Cycles were repeated at 2 -week intervals until disease progression or unacceptable toxicity developed. Treatment with a $5 \mathrm{HT}_{3}$ receptor antagonist was recommended to control nausea and vomiting. All treatments were administered via implantable ports and using disposable pumps (Baxter Corporation).

\section{Dose modification}

Toxicities were graded according to the NCI CTC version 2.0, 30 January 98, except for oxaliplatin-induced neurosensory toxicity, for which a specific grading scale was used. This scale was a combination of the NCI CTC and the Levi et al scale (Levi et al, 1992).

Doses of oxaliplatin and 5-FU were modified in a stepwise fashion according to a specific algorithm in the case of febrile neutropenia, ANC $<0.5 \times 10^{9} 1^{-1}$, platelet nadir $<50 \times 10^{9} 1^{-1}$ or a 1 -3-week dose delay due to prolonged ANC, or platelet, recovery. Initially, the bolus dose of 5-FU was omitted from subsequent cycles; then, if the toxicity recurred, the 5-FU infusional dose was reduced to $2 \mathrm{~g} \mathrm{~m}^{-2}$ for subsequent cycles, and then the oxaliplatin dosage was reduced to $75 \mathrm{mg} \mathrm{m}^{-2}$ for subsequent cycles. Since 5FU has previously been shown to contribute more to neutropaenia and since the bolus dose was the most convenient component to modify it was chosen first, followed by the infusional component. This strategy was subsequently also chosen by the French group in designing the Folfox7 regimen (Maindrault-Goebel et al, 2001). Treatment could be recommenced once febrile neutropenia resolved or the ANC improved to $\geqslant 1.5 \times 10^{9} 1^{-1}$ or the platelet count improved to $\geqslant 100 \times 10^{9} 1^{-1}$. If the toxicities recurred after the described 5-FU and oxaliplatin dose modifications, or a dose was delayed $>2$ weeks due to toxicity, study therapy was discontinued. No dose reductions were planned for changes in haemaglobin or total white blood cell concentrations.

Neurological symptoms were assessed according to a specific Neurosensory Toxicity Scale, which rated neurological symptoms Grade 1-4 according to their persistence, degree of functional impairment and impact on activities of daily living (ADL). Grade 1 described neurological symptoms that resolved and did not interfere with function, grade 2 symptoms interfered with function but not ADL, grade 3 toxicity indicated pain or functional impairment that interfered with ADL and grade 4 denoted persistent symptoms that were disabling or life threatening.

If patients experienced grade 2 or 3 neurosensory toxicity, oxaliplatin and 5-FU were both withheld until symptoms resolved and then restarted at a reduced dose of $75 \mathrm{mg} \mathrm{m}^{-2}$. If symptoms recurred, oxaliplatin was again withheld until resolution and then restarted at a reduced dose of $50 \mathrm{mg} \mathrm{m}^{-2}$. If symptoms recurred after two dose reductions, or persisted after a dose delay of $>2$ weeks, treatment was discontinued due to unacceptable neurosensory toxicity. Failure of neurotoxicity to return to grade I necessitated removal from the study. If pseudo-laryngopharyngeal dysesthesiae occurred, subsequent doses of oxaliplatin were administered as a 6-h infusion.

\section{Study parameters}

Assessments for haematological parameters and biochemistry were performed every 2 weeks prior to each cycle and patients were questioned for symptoms of neurosensory toxicity prior to each oxaliplatin dose.

All tumours were measured by the same imaging method used at baseline for tumour size. A measurable tumour was defined as a mass with clearly defined margins and could be clearly measured on physical exam, X-ray or other radiograph with a ruler or caliper. At least one diameter had to be $\geqslant 2 \mathrm{~cm}$ on radiographic imaging. Lesions with all dimensions $<1 \mathrm{~cm}$ were omitted. 
Responses were assessed every four cycles (8 weeks). Complete and partial responses were confirmed by repeat tumour evaluations at least 4 weeks later. Standard definitions and standard criteria for bidimensional measurement were used according to WHO (Miller et al, 1981) in assessing responses. Stable disease (SD) was confirmed after 4 weeks and had to last a minimum of 16 weeks from the commencement of therapy.

Progression-free survival was measured from the date of commencing protocol treatment to the date of first progression or death from any cause without progression. Overall survival was measured from the date of commencing protocol treatment to the date of death from any cause.

\section{Statistical considerations}

Confidence intervals (CO) (95\%) for the response rates were estimated using the exact probabilities of the binomial distribution.

The Kaplan-Meier method was used to calculate PFS and OS.

Statistical analyses were performed using StatXact procedures. (StatXact 4.0.1. Cytel Software Corporation, Cambridge, MA, USA, 1999).

Dose intensity was defined as the actual dose intensity relative to the protocol dose intensity. The initial doses of the drugs used were calculated using BSA in $\mathrm{m} 2=\exp (0.425 \times \ln ($ weight $)+$ $0.725 \times \ln ($ height $)-4.9358)$ to assess whether initial doses were given as per protocol. The relative dose intensities were calculated for each drug by dividing the total dose given as a proportion of the planned protocol dose by the total number of days on treatment relative to protocol time. The protocol time was 14 days for each cycle given. The total number of days on treatment was calculated from day 1 of the first cycle to 14 days after day 1 of the last cycle.

\section{RESULTS}

\section{Patient characteristics}

A total of 62 patients were registered from four Australian centres between September 1999 and October 2000. In all, 61 patients received $\geqslant$ one dose of oxaliplatin and were analysed for treatment and safety results. Pretreatment characteristics of these patients are listed in Table 1 . While the median age was 61 years (range $31-$ 76 ), $59 \%$ were aged 60 years or older, $21 \%$ were over $70,97 \%$ were Caucasian.

Six of the 62 registered patients were deemed ineligible; one who had received adjuvant treatment less than 6 months prior to study

Table I Patient characteristics at registration (total 61 patients who received $\geqslant$ | oxaliplatin dose)

\begin{tabular}{lcc}
\hline & No. & $\%$ \\
\hline Sex & 45 & 74 \\
$\quad$ Male & 16 & 26 \\
Female & & \\
ECOG performance status & & 48 \\
0 & 29 & 39 \\
I & 24 & 13 \\
2 & 8 & 66 \\
Primary site & & 34 \\
Colon & & \\
Rectum & 40 & 67 \\
Prior adjuvant therapy for colorectal cancer & 21 & 33 \\
No & & \\
Yes & 41 & \\
\hline
\end{tabular}

entry, two with insufficient measurable disease, one who had received prior radiosensitising chemotherapy for advanced disease and two with laboratory parameters outside the eligible range on the days of treatment. The remaining 56 eligible patients were analysed for efficacy.

At the close-out date of 22 May 2003, the median potential follow-up time from the commencement of treatment was 37 months (range 31-44 months).

\section{Treatment given}

In total, 587 cycles were given, with a range of $1-20$ cycles and a median of 112 -weekly cycles per patient.

Out of 61 patients, 58 received more than one cycle of therapy. Of these, 46 (79\%) experienced a dose reduction of more than $10 \%$ relative to the dose in cycle 1 for at least one drug. In all, 26 patients $(45 \%)$ had their oxaliplatin dose reduced and one patient (2\%) had their leucovorin dose reduced by $>10 \%$ on at least one cycle as an unspecified administrative error. A total of 44 patients (76\%) had their 5-FU bolus dose and 27 (47\%) had their 5-FU infusion dose reduced by $>10 \%$ on at least one cycle.

Although the protocol specified 14 days between cycles, only eight patients $(14 \%)$ received all their treatment cycles within \pm 2 days of the protocol 14-day period. In total, 136 cycles (23\%) in 50 patients were delayed more than 2 days. Of these, 116 cycles $(20 \%)$ were delayed for toxicity reasons.

\section{Objective tumour responses}

Of the 56 eligible patients, the best response to treatment was assessed as a complete response (CR) in three patients (5\%) and a partial response (PR) in 26 patients $(46 \%)$, for an overall objective response rate of $52 \%(95 \%$ CI $38-65 \%)$. The full response data are shown in Table 2.

\section{Progression-free survival (PFS)}

By the close-out date, four patients (7\%) were alive without progression (two have since died) and 52 patients (93\%) had progressed (two patients died without objective evaluation of their progression). The estimated median PFS was 8.2 months (95\% CI 7.1-9.9 months) with an estimated proportion of patients surviving without progression of $30 \%(95 \%$ CI $20-44 \%)$ at 1 year and $11 \%(5-22 \%)$ at 2 years (Figure 2 ).

\section{Survival}

As at the close-out date, $10(18 \%)$ patients were still alive and 46 $(82 \%)$ patients had died, $45(80 \%)$ due to progressive disease and

Table 2 Best objective response to treatment

\begin{tabular}{lcc}
\hline & \multicolumn{2}{c}{ Total (56) } \\
\cline { 2 - 3 } & No. & $\%$ \\
\hline Complete response (CR) & 3 & 5 \\
Partial response (PR) & 26 & 46 \\
Stable disease (SD) & 10 & 18 \\
Progressive disease (PD) & $9(12)^{\mathrm{a}}$ & $16(21 \%)^{\mathrm{a}}$ \\
Not assessable (NA) & $8(5)^{\mathrm{a}}$ & $14(9 \%)^{\mathrm{a}}$ \\
\hline
\end{tabular}

Three patients were not assessable by our criteria, but displayed clinical disease progression that was not objectively validated (two received only two treatment cycles before clinical progression and one received four cycles and only one assessment before clinical progression). ${ }^{b}$ Not assessable patients were one patient who had clinical disease progression after two cycles but was too unwell to repeat imaging, five patients who received fewer than planned cycles of therapy, one patient whose scans were lost and was thus not assessed properly at cycle 7, and one patient who received only one assessment before clinical progression. 
one $(2 \%)$ during surgery for complications of colorectal cancer. The estimated median OS was 18.7 months (95\% CI 14.0-23.4 months), with an estimated proportion of patients surviving of $73 \%(95 \%$ CI $60-83 \%)$ at 1 year and $34 \%(23-47 \%)$ at 2 years (Figure 3).

\section{Dose intensity}

The median relative dose intensity of oxaliplatin was $83 \%$, range $39-103 \%$, of LV $88 \%$, range $39-102 \%$, of bolus $5-\mathrm{FU} 50 \%$, range $0-102 \%$ (omitted for haematologic or gastrointestinal toxicity) and of 5-FU infusion $83 \%$, range $35-103 \%$. In all, 55\% of patients received $100+/ 5 \%$ of oxaliplatin for all cycles given see Figure 4.

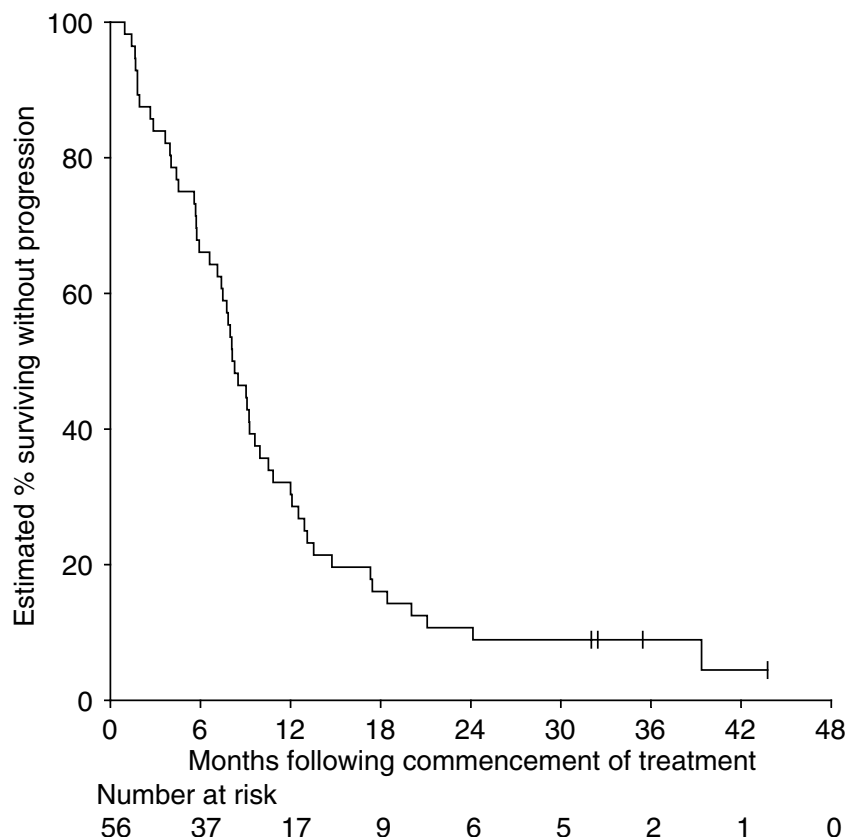

Figure 2 Progression-free survival. Ticks indicate censored survival times.

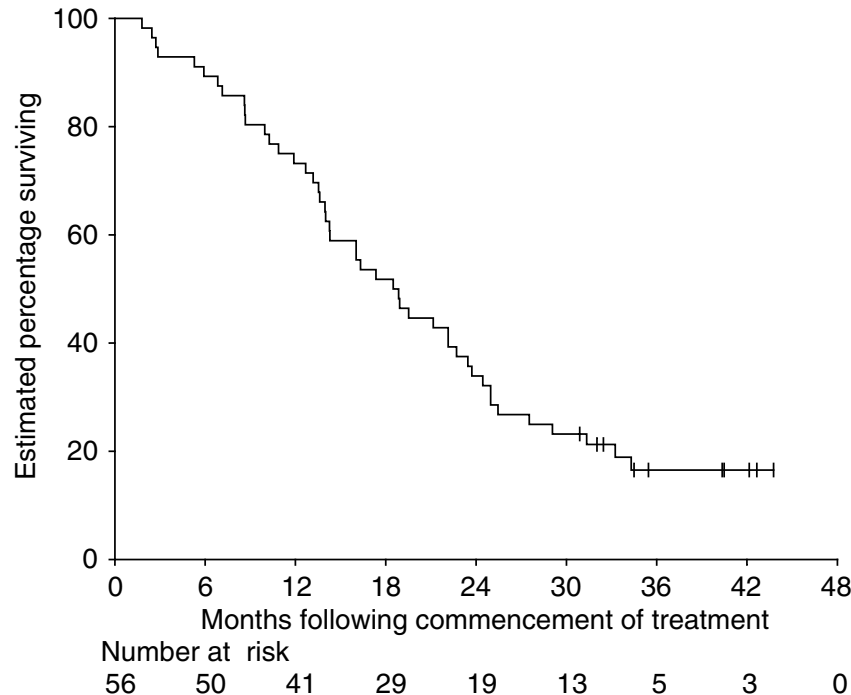

Figure 3 Overall survival. Ticks indicate censored survival times.

\section{Toxicity}

At the study close-out date, of the 61 patients who received $\geqslant$ one treatment cycle, two patients $(3 \%)$ had completed 12 treatment cycles and 59 patients (97\%) had discontinued treatment. In all, 16 patients $(26 \%)$ discontinued due to disease progression or death, $25(41 \%)$ due to toxicity $(15(25 \%)$ neurotoxicity, $6(10 \%)$ haematological toxicity and four (7\%) due to other toxicity), 14 (23\%) due to a decision of the patient or investigator and four (7\%) due to other reasons.

Grade 2, 3 and 4 acute toxicities are listed in Tables 3 and 4 . The most commonly reported toxicity was neutropenia. Grade 3/4 neutropenia occurred in $22(36 \%)$ patients; however, only three patients $(5 \%)$ experienced febrile neutropenia. There were no toxic deaths.

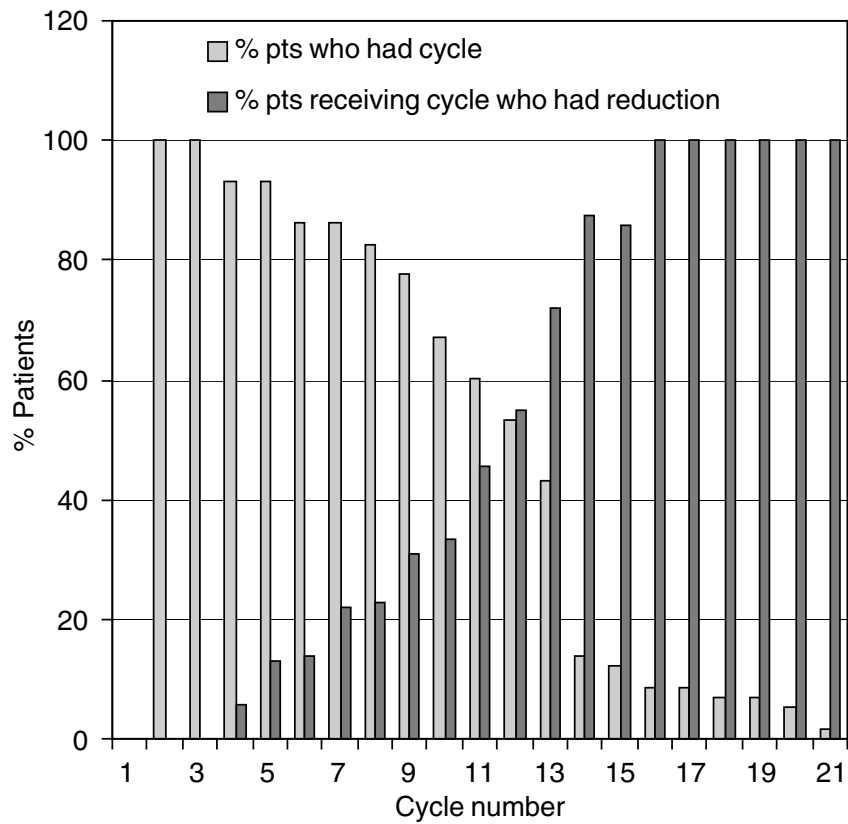

Figure 4 Dose intensity of oxaliplatin by cycle.

Table 3 Haematological toxicities (Worst grade while on treatment)

\begin{tabular}{lccc}
\hline & \multicolumn{3}{c}{ Total = 6 I } \\
\cline { 2 - 4 } & $\begin{array}{c}\text { Grade 2 } \\
\text { No. (\%) }\end{array}$ & $\begin{array}{c}\text { Grade 3 - } \\
\text { No. (\%) }\end{array}$ & $\begin{array}{c}\text { Grade 4 - } \\
\text { No. (\%) }\end{array}$ \\
\hline Thrombocytopenia & $9(15)$ & $4(7)$ & $0(0)$ \\
Neutropenia & $18(30)$ & $17(28)$ & $5(8)$ \\
Febrile neutropenia & $3(5)$ & & \\
\hline
\end{tabular}

Table 4 Nonhaematological toxicities

Total $=61$

Grade 2 - No. (\%) Grade 3 - No. (\%) Grade 4 - No. (\%)

\begin{tabular}{lccl}
\hline Diarrhoea & $12(20)$ & $7(\mathrm{II})$ & $0(0)$ \\
Mucositis & $7(1 \mathrm{I})$ & $2(3)$ & $0(0)$ \\
Vomiting & $7(1 \mathrm{I})$ & $2(3)$ & $0(0)$ \\
Neurological & $20(33)$ & $13(2 \mathrm{I})$ & $0(0)$ \\
Fatigue & $3(5)$ & $2(3)$ & $0(0)$ \\
Nausea & $6(10)$ & $2(3)$ & $0(0)$ \\
\hline
\end{tabular}


Neurological toxicity was also common with 20 patients (33\%) experiencing grade 2 and 13 patients (21\%) experiencing grade 3 neurotoxicity during or post treatment. No grade 4 neurotoxicity was reported.

For patients with grade $2 / 3$ neurotoxicity, the median time to recovery to grade $0 / 1$ after ceasing treatment was estimated via the Kaplan - Meier method to be 3.9 months (95\% CI 0-6.7 months). It was also estimated that $36 \%(21-54 \%)$ and $21 \%(10-40 \%)$ had persisting grade 2 or 3 neurotoxicity at 6 and 12 months, respectively. For grade 3 neurotoxicity specifically, it was estimated that $71 \%$ of the patients had recovered 12 months after ceasing treatment.

Two patients experienced a worsening of their neurological toxicity from grade 1 to 2 and one patient reported grade 3 myopathy after ceasing treatment.

\section{Hospitalisations during treatment}

Nine patients (15\%) required hospitalisation for toxicity; two patients for febrile neutropenia, one patient with fever and rigours without neutropenia, two patients with nausea and vomiting (including one patient with additional abdominal pain), one with diarrhoea (grade 3 ) and dehydration (grade 2), one for investigation of chest pain, one with porta-cath site erythema to investigate infection, and one with candidiasis and elevated blood sugar.

A total of $19(31 \%)$ of patients were hospitalised for other reasons, not related to toxicity.

\section{DISCUSSION}

The combination of oxaliplatin and 5-FU/LV has proven to be a major advance in the treatment of colorectal cancer both in the metastatic and now adjuvant setting (André et al, 2004). Studies with successive FOLFOX regimens are attempting to define the optimal oxaliplatin/5-FU/LV dose schedule. Important considerations include the haematological toxicity, a recognised side effect of both oxaliplatin and 5-FU treatment, and the cumulative neurotoxicity, which is dictated by oxaliplatin treatment duration.

In our study, we used a modified FOLFOX6 regimen, which focused upon maximising oxaliplatin dose intensity and improved patient convenience by allowing all patients to be treated as outpatients and reducing the number of outpatient visits. The response rate of $52 \%$ and the median PFS and OS of 8.2 and 18.7 months, respectively, is at the upper end of those reported in randomised trials of FOLFOX4 as front-line therapy, and similar to that of FOLFOX6 (Tournigand et al, 2004) in previously untreated metastatic colorectal cancer.

This is the first report of this modification to the FOLFOX6 regimen in untreated patients and the only report of this simplified FOLFOX regimen in either pretreated or untreated patients outside Europe. As such, it confirms a modification that is more convenient than FOLFOX4 and appears to have at least comparable, if not enhanced, efficacy, due to the increased oxaliplatin dose. In addition, there was neither a major increase in neurotoxicity or haematological toxicity compared to FOLFOX4 in the metastatic setting.

One of the two issues identified in the analysis of phase II studies of different FOLFOX combinations was the hypothesis that the dose intensity of oxaliplatin is important. Early studies with folfox 2 at $100 \mathrm{mg} \mathrm{m}^{-2}$ showed a high response rate (46\%) but high neurotoxicity. Subsequently, reduction in oxaliplatin dose to $85 \mathrm{mg} \mathrm{m}^{-2}$ in Folfox4, lowered toxicity but compromised efficacy $(24 \%)$, with a dose intensity of oxaliplatin of $74 \mathrm{mg} \mathrm{m}^{-2}$. (reviewed in Maindrault-Goebel et al, 2000). Thus, in FOLFOX6, the dose of oxaliplatin was increased from 85 to $100 \mathrm{mg} \mathrm{m}^{-2}$. However, in the phase II trials with this regimen, dose intensity remained reduced compared to Folfox2, where $89 \%$ of patients had $>85 \mathrm{mg} \mathrm{m}^{-2}$ vs only 59\% for Folfox6. In Folfox 6 second line, the associated response rate also remained similar to Folfox4, possibly due to oxaliplatin dose modification for haematological toxicity (MaindraultGoebel et al, 1999). This led to a redesigned schedule, FOLFOX7, which increased the dose of oxaliplatin further to $130 \mathrm{mg} \mathrm{m}^{-2}$, but reduced the total number of doses administered and focused on 5-FU dose reductions in response to nonneurological toxicity. In a phase II study in relapsed patients, FOLFOX7 produced a response rate of $42 \%$ and grade $3 / 4$ neurological toxicity of 15\% (Maindrault-Goebel et al, 2001). A subsequent study showed that a modified 5-FU schedule combined with $85 \mathrm{mg} \mathrm{m}^{-2}$ but with reductions of both oxaliplatin and 5-FU for haematologic toxicity once again reducing the oxaliplatin dose, was associated with marked reduction in efficacy in the second line setting $-12 \%$ in 37 patients but with a remarkable $72 \%$ response rate in 25 first-line patients (Cheeseman et al, 2002). The discrepancy remains unexplained, but still suggests that the need for enhanced dose intensity of oxaliplatin above $85 \mathrm{mg} \mathrm{m}^{-2}$ requires exploration.

In our study, we chose to focus on adjusting the 5-FU ahead of oxaliplatin and were able to achieve an impressive response rate and maintain oxaliplatin dose intensity similar to FOLFOX7 (85\% with dose intensity $>100 \mathrm{mg} \mathrm{m}^{-2}$ in first four cycles in Folfox7 Maindrault-Goebel et al (2001) vs 93\% in ours), but without needing to increase the oxaliplatin dose further above $100 \mathrm{mg} \mathrm{m}^{-2}$. A potential benefit of our schedule lies in the issue of neurotoxicity.

Oxaliplatin-induced neurotoxicity consists of an acute rapidonset sensory neuropathy, often triggered by cold or sudden temperature changes, and a chronic cumulative sensory neuropathy that occurs after several cycles of treatment, and is potentially dose limiting. In De Gramont's et al (2000) phase III study, $18 \%$ of patients experienced grade 3 sensory neurotoxicity; however, $74 \%$ of patients experienced a reversal of neurotoxicity after treatment was discontinued. In our study, $21 \%$ of patients experienced grade 3 neurotoxicity but with only a limited residual effect, as at 12 months $71 \%$ of patients had recovered. In the randomised FOLFOX6 study, $34 \%$ had grade 3 toxicity. Similarly, in the recent MOSAIC study (André et al, 2004), grade 3 neurotoxicity was observed in $12 \%$ of patients, with $94 \%$ of patients experiencing a partial or total recovery within 6 months of stopping treatment. This is summarised in Table 5. Since the data suggest that cumulative dose, rather than dose intensity, leads to neurotoxicity, it follows that patients in our study would be allowed an increased number of treatment cycles compared with FOLFOX7.

An alternative approach to dealing with neurotoxicity is currently being tested by the French group in the Optimox studies (De Gramont et al, 2004), with previously untreated patients being randomised to FOLFOX4 until progression, or FOLFOX7 given for six cycles followed by a simplified LV5FU2 schedule every 2 weeks for 12 cycles. FOLFOX7 is then reintroduced for six cycles, or earlier in case of progression, in patients who have a response or $\mathrm{SD}$ at the first FOLFOX administration. From the preliminary results in 623 patients, the authors have concluded that the FOLFOX7 arm has a response rate and toxicity similar to the FOLFOX4 regimen and is more convenient. Only further follow-up will establish the role of shorter more intensive regimens $v s$ the established FOLFOX approach. An intermediate regimen that maximises oxaliplatin dose intensity by focusing on 5-FU dose modification and preserving oxaliplatin dose may be equally valid.

The recent MOSAIC data (André et al, 2004) suggest that FOLFOX4 has a major role to play in the adjuvant setting, where enhanced efficacy with increased oxaliplatin dose intensity and improved convenience are even more important issues. The recent use of capecitabine combined with oxaliplatin improves convenience and has shown promising efficacy and tolerability data (Cassidy et al, 2004). This has led to a current randomised trial to 


\begin{tabular}{|c|c|c|c|c|c|c|c|}
\hline Regimen (ref.) & $\begin{array}{c}\text { Regimen } \\
\text { no. }\end{array}$ & $\begin{array}{l}\text { PFS } \\
\text { mos }\end{array}$ & $\begin{array}{l}\text { Planned dose } \\
\mathrm{mg} \mathrm{m}^{-2}\end{array}$ & $\begin{array}{l}\text { Received dose } \\
\text { intensity }\end{array}$ & $\begin{array}{c}\text { Haematologic } \\
\text { toxicity } \\
\text { Grade } 3 / 4(\%)\end{array}$ & $\begin{array}{l}\text { Neuro Tox. } \\
\text { Grade } 3 \text { (\%) }\end{array}$ & $\begin{array}{c}\text { Resp. rate } \\
\text { (\%) }\end{array}$ \\
\hline Folfox $N=46$ (Maindrault-Goebelt et al, 2000) & 2 & 7 & 100 & 0.98 & 39 & 33 & $46^{*}$ \\
\hline Folfox $N=40$ (Maindrault-Goebelt et al, 2000) & 3 & 6 & 85 & 0.79 & 15 & 28 & $20^{*}$ \\
\hline Folfox $N=57$ (Andre et al, 2003) & 4 & 5.1 & 85 & 0.89 & 37 & 16 & $24^{*}$ \\
\hline Folfox $N=210$ (De Gramont et al, 2000) & 4 & 9.0 & 85 & 0.86 & 42 & 18 & $49^{*}$ \\
\hline Folfox $N=60$ (Maindrault-Goebelt et al, 2000) & 6 & 5.3 & 100 & 0.86 & 24 & 16 & $27^{*}$ \\
\hline Folfox $N=1||$ (Tournigand et al, 2004) & 6 & 8.0 & 100 & 0.85 & 44 & 34 & $54^{*}$ \\
\hline Folfox $N=48$ (Maindrault-Goebelt et al, 2000) & 7 & & 130 & 0.85 & 9 & 27 & $42^{*}$ \\
\hline Cheeseman $N=25$ (Cheeseman et al, 2002) & & 10.6 & 85 & NS & 4 & $0^{\dagger}$ & $72^{*}$ \\
\hline Goldstein $N=61$ & & 8.2 & 100 & 0.83 & 36 & 21 & $51^{*}$ \\
\hline
\end{tabular}

${ }^{*}=$ Second line Therapy; ${ }^{\dagger}=$ Only assessed for first six cycles so not comparable.

examine if the capecitabine combination is equivalent to FOLFOX4, if it is, then attempts to increase oxaliplatin dose intensity in conjunction with capecitabine may be indicated. In the interim, this intermediate modification, with a higher dose of oxaliplatin and an entirely outpatient regimen, appears active, convenient and safe. Our focus on avoiding oxaliplatin dose reductions for haematological toxicity increased oxaliplatin dose intensity, and possibly improved efficacy, without unacceptable neurologic outcomes. As such, it may be recommended for use in the metastatic setting to enhance patient acceptability of this highly active treatment approach while awaiting evidence to support the routine use of capecitabine and the addition of targeted therapies such as bevacucimab and cetuximab.

\section{ACKNOWLEDGEMENTS}

We thank Liz Chatwin for editorial assistance, Jennifer Smith (Centre for Biostatistics and Clinical Trials, PMCC) for statistical expertise, all the research nurses and study coordinators, and most of all the patients who gave us their time and their enthusiasm. This study was sponsored by Sanofi $\sim$ Synthelabo.

\section{REFERENCES}

André T, Boni C, Mounedji-Boudiaf L, Navarro M, Tabernero J, Hickish T, Topham C, Zaninelli M, Clingan P, Bridgewater J, TabahFisch I, de Gramont A (2004) Oxaliplatin, fluorouracil, and leucovorin as adjuvant treatment for colon cancer. $N$ Engl $J$ Med 350: $2343-2351$

Cassidy J, Tabernero J, Twelves C, Brunet R, Butts C, Conroy T, Debraud F, Figer A, Grossmann J, Sawada N, Schoffski P, Sobrero A, Van Cutsem E, Diaz-Rubio E (2004) XELOX (capecitabine plus oxaliplatin): active firstline therapy for patients with metastatic colorectal cancer. J Clin Oncol 22(11): 2084-2091

Cheeseman SL, Joel SP, Chester JD (2002) A 'modified de Gramont' regimen of fluorouracil, alone and with oxaliplatin, for advanced colorectal cancer. Br J Cancer 87(4): 393-399

De Gramont A, Cervantes A, Andre T, Fiiier A, Ledo GI, Fiesch M, Mineur I, Russ G, Quinaux E, Etienne P-L (2004) OPTIMOX study: FOLFOX 7/ LV5FU2 compared to FOLFOX 4 in patients with advanced colorectal cancer. J Clin Oncol 22(14S): 3525 (2004 ASCO Annu Meet Proc)

De Gramont A, Figer A, Seymour M, Homerin M, Hmissi A, Cassidy J, Boni C, Cortes-Funes H, Cervantes A, Freyer G, Papamichael D, Le Bail N, Louvet C, Hendler D, de Braud F, Wilson C, Morvan F, Bonetti A (2000) Leucovorin and fluorouracil with or without oxaliplatin as first-line treatment in advanced colorectal cancer. J Clin Oncol 18(16): $2938-2947$

Giacchetti S, Perpoint B, Zidani R, Le Bail N, Faggiuolo R, Focan C, Chollet P, Llory JF, Letoumeau Y, Coudert B, Bertheaut-Cvitkovic F, LarregainFournier D, Le Rol A, Walter S, Adam R, Misset JL, Levi F (2000) Phase III multicentre randomized trial of oxaliplatin added to chronomodulated fluorouracil-leucovorin as first-line treatment of metastatic colorectal cancer. J Clin Oncol 18: 136-147

Goldberg RM, Sargent DJ, Morton RF, Fuchs CS, Ramanathan RK, Williamson SK, Findlay BP, Pitot HC, Alberts SR (2004) A randomized controlled trial of fluorouracil plus leucovorin, irinotecan, and oxaliplatin combinations in patients with previously untreated metastatic colorectal cancer (see comment). J Clin Oncol 22: 23-30
Levi F, Misset JL, Brienza S, Adam R, Metzger G, Itzakhi M, Caussanel JP, Kunstlinger F, Lecouturier S, Descorps-Declere A (1992) A chronopharmacologic Phase II clinical trial with 5-fluorouracil, folinic acid, and oxaliplatin using an ambulatory multichannel programmable pump. Cancer 69: 893-900

Maindrault-Goebel F, de Gramont A, Louvet C, Andre T, Carola E, Gilles V, Lotz JP, Toumigand C, Mabro M, Molitor JL, Artru P, Izrael V, Krulik M (2000) Evaluation of oxaliplatin dose intensity in bimonthly leucovorin and 48- hour 5-fluorouracil continuous infusion regimens (FOLFOX) in pretreated metastatic colorectal cancer. Oncology Multidisciplinary Research Group (GERCOR). Ann Oncol 11: 1477-1483

Maindrault-Goebel F, de Gramont A, Louvet C, Andre T, Carola E, Mabro M, Artru P, Gilles V, Lotz JP, Izrael V, Krulik M, Oncology Multidisciplinary Research Group (GERCOR) (2001) High-dose intensity oxaliplatin added to the simplified bimonthly leucovorin and 5fluorouracil regimen as second-line therapy for metastatic colorectal cancer (FOLFOX 7). Eur J Cancer 37: 1000-1005

Maindrault-Goebel F, Louvet C, Andre T, Carola E, Lotz JP, Molitor JL, Garcia ML, Gilles-Amar V, Izrael V, Krulik M, de Gramont A (1999) Oxaliplatin added to the simplified bimonthly leucovorin and 5fluorouracil regimen as second-line therapy for metastatic colorectal cancer (FOLFOX6). GERCOR Eur J Cancer 35: 1338-1342

Miller AB, Hoogstraten B, Staquet M, Winkler A (1981) Reporting results of cancer treatment. Cancer 47: 207-214

Raymond E, Buquet-Fagot C, Djelloul S, Mester J, Cvitkovic E, Allain P, Louvet C, Gespach C (1997) Antitumor activity of oxaliplatin in combination with 5- fluorouracil and the thymidylate syntiiase inhibitor AG337 in human colon, breast and ovarian cancers. Anticancer Drugs 8(9): $876-885$

Tournigand C, Andre T, Achille E, Lledo G, Flesh M, Mery-Mignard D, Quinaux E, Couteau C, Buyse M, Ganem G, Landi B, Colin P, Louvet C, de Gramont A (2004) FOLFIRI followed by FOLFOX6 or the reverse sequence in advanced colorectal cancer: a randomized GERCOR study. J Clin Oncol 22: 229-237 